Research Article

\title{
Gray Relation Analysis for Optimal Selection of Bridge Reinforcement Scheme Based on Fuzzy-AHP Weights
}

\author{
Yanbin Tan, ${ }^{1}$ Zhe Zhang, ${ }^{1}$ Huili Wang $\mathbb{D}^{1,2}$ and Shaobo Zhou ${ }^{1}$ \\ ${ }^{1}$ National \& Local Joint Engineering Laboratory of Bridge and Tunnel Technology, Dalian University of Technology, \\ Dalian 116023, China \\ ${ }^{2}$ State Key Laboratory of Structural Analysis for Industrial Equipment, Dalian University of Technology, Dalian 116023, China
}

Correspondence should be addressed to Huili Wang; wanghuili@dlut.edu.cn

Received 16 September 2020; Accepted 6 July 2021; Published 23 July 2021

Academic Editor: Yung-Chung Wang

Copyright ( $\odot 2021$ Yanbin Tan et al. This is an open access article distributed under the Creative Commons Attribution License, which permits unrestricted use, distribution, and reproduction in any medium, provided the original work is properly cited.

In order to solve the problem on optimal selection of old bridge reinforcement schemes, a decision-making method of gray relation analysis based on fuzzy-AHP weights is proposed. Firstly, the fuzzy-AHP is used to develop the decision index system of old bridge reinforcement schemes and determine the weight of decision indexes. The 0.1-0.9 scale method is introduced as the index judgment criterion, and the weight judgment matrix is established. Through the consistency test, the relative weight vector of each decision index in the index layer is calculated. Secondly, according to the gray relation model of the old bridge reinforcement schemes, the decision matrix is constructed, and the gray relation coefficient matrix is calculated to obtain the gray relation coefficient corresponding to the ideal optimal scheme. Finally, the optimal scheme is determined. Through an engineering example, the reinforcement scheme of a concrete-filled steel tube arch bridge deck system is calculated and analyzed, and the best reinforcement scheme is selected. The optimal selection result is consistent with the actual reinforcement scheme available for the bridge. The decision-making method of gray relation analysis based on fuzzy-AHP weights make the evaluation system more organized and systematic and the index weight more operable and quantitative, reduce the subjective evaluation impact, and make the evaluation result more objective and reliable. Considering the fuzzy and gray information of comparison and selection, the optimal scheme with high feasibility and applicability is selected by the gray relation method.

\section{Introduction}

With the operation of in-service bridges, due to the increase of traffic volume and vehicle load, the influence of unfavorable factors in the surrounding environment, and the natural aging of materials, the bridge structure is faced with performance degradation during its life cycle, resulting in the weakening of its function. In order to meet the traffic development needs, ensure the safety of bridge structure, satisfy the use function, and extend the service life, it is necessary to reconstruct and reinforce the old bridges. Many bridge reinforcement methods are now available, such as reinforcing the main girder cross section, reducing the dead load, and changing the structural system. These methods comprehensively consider the bearing capacity, durability, impact of traffic interruption, economic rationality of reinforcement cost, and complexity of reinforcement technology. The determination of the reinforcement scheme is the key to the success of the reconstruction and reinforcement, especially the feasibility, reliability, and economy of the reinforcement scheme. The optimal selection process of the old bridge reinforcement scheme is a multiobjective decision-making process. The multiobjective decision-making is characterized by the conflicting objectives, inconsistent objective dimensions, and adjustable "optimal solution," making the decisionmaking process more complicated [1]. Dağdeviren and Yüksel developed the evaluation index system of bridge reinforcement schemes based on the analytic hierarchy process (AHP) and performed the optimal selection analysis [2]. Nguyen et al. established a two-level fuzzy optimal selection model and used the nonstructural fuzzy 
decision-making theory for the bridge scheme comparison and selection [3]. Maghrabie et al. proposed a multilayer and multiobjective fuzzy optimal selection model and applied it to the comparison and optimal selection of bridge schemes [4]. Thakur and Ramesh combined the AHP method and the entropy method to weigh the evaluation indexes and established the gray relation method based on the combined weight for the optimal selection of bridge reinforcement schemes [5]. $\mathrm{Li}$ and Chen proposed to improve the fuzzy belief structure method to determine the weight and established the gray relation optimal selection model for the optimal selection research of bridge reinforcement schemes [6]. Kalemci et al. used the combined weight method to establish a simplified gray wolf optimization algorithm method model for the optimal selection of bridge reinforcement schemes [7]. For this reason, according to the characteristics of the old bridge reinforcement scheme evaluation, this paper organically combines the fuzzy theory [8-11] with AHP, proposes a method to determine the index weight, and adopts the gray relation method [12] to realize the comprehensive evaluation of each reinforcement alternative and thus the selection of optimal scheme, making the optimal selection process more simple, objective, reasonable, and reliable. Therefore, it is necessary to carry out the optimal selection of bridge reinforcement schemes in order to achieve the basic objectives in technical feasibility, reliability, economic rationality, construction simplicity, and quality assurance.

In this paper, four bridge reinforcement schemes for an old bridge are carried out. Many factors need to be considered, such as the aging and disease degree of the original structure, the technical feasibility, and the impact of traffic interruption, which are more risky and difficult compared with the new bridge construction. In order to solve the problem on optimal selection of old bridge reinforcement schemes, a decision-making method of gray relation analysis based on fuzzy-AHP weights is proposed.

\section{Evaluation Index System}

Since several optimal selection indexes need to be comprehensively considered in the decision-making process for optimal selection of old bridge reinforcement schemes, the fuzzy-AHP [13] shall be firstly used to organize and hierarchize the optimal selection problem, thus developing a hierarchy-based optimal selection index system, as shown in Table 1. The system is divided into three layers: the first layer mainly includes the optimal selection purpose or desirable result, that is, to determine the optimal reinforcement scheme; the second layer includes the subordinate indexes of the upper layer, which represent the optimal selection index factors to be satisfied before the optimal selection purpose is achieved; the third layer is the basic index layer, which includes the most basic decomposition indexes of the optimal selection problem and directly reflects the comprehensive attribute information of each reinforcement scheme.

\section{Decision Index Weight Determined by Fuzzy- AHP}

3.1. Construction of Fuzzy Complementary Judgment Matrix. Based on the principle of fuzzy-AHP, the mutual priority relationship among the decision indexes is determined, and the $0.1-0.9$ scale method [14] is used as the judgment criterion for the pairwise comparison of the indexes to establish the weight judgment matrix $C=\left(c_{i j}\right)_{n \times n}$ :

$$
C=\left[\begin{array}{cccc}
c_{11} & c_{12} & \ldots & c_{1 n} \\
c_{21} & c_{22} & \ldots & c_{2 n} \\
\ldots & \ldots & & \ldots \\
c_{n 1} & c_{n 2} & \ldots & c_{n n}
\end{array}\right],
$$

where $c_{i j}$ is the relative weight vector based on the $j$ th decision index than the $i$ th decision.

3.2. Consistency Check of Judgment Matrix. The weight vector of matrix $C$ is obtained by solving the judgment matrix with the formula derived in [15] $W=\left[\begin{array}{llll}w_{1} & w_{2} & \cdots & w_{n}\end{array}\right]^{T}:$

$$
w_{i}=\frac{\sum_{j=1}^{n} c_{i j}+(n / 2)-1}{n(n-1)} \text {. }
$$

Set

$$
w_{i j}=\frac{w_{i}}{w_{i}+w_{j}}, \quad(i, j=1,2, \ldots, n) .
$$

Then, the $N$-order matrix $S=\left(w_{i j}\right)_{n \times n}$ is taken as the characteristic matrix of the judgment matrix $C$. The compatibility index of $C$ and $S$ is

$$
I(C, S)=\frac{1}{n^{2}} \sum_{i=1}^{n} \sum_{j=1}^{n}\left|c_{i j}+w_{i j}-1\right| .
$$

With regard to the attitude $\alpha$ of the decision maker, when the compatibility index $I(C, S) \leq \alpha$, the judgment matrix is considered to be satisfactorily consistent. The smaller the $\alpha$, the higher the consistency requirement for the matrix, which is generally taken as $\alpha=0.2$.

Assuming there are $k$ experts, then the fuzzy judgment matrix $C_{k}=\left(c_{i j}^{k}\right)_{n \times n}$; when $k=1,2, \ldots, t$, the corresponding weight vector set $W_{k}=\left[\begin{array}{llll}W_{1} & W_{2} & \ldots & W_{t}\end{array}\right]$ [15], and the characteristic matrix $S_{k}=\left(w_{i j}^{k}\right)_{n \times n}$. If $t$ judgment matrices $B(k)$ and other judgment matrices are of satisfactory consistency, the fuzzy-AHP weight is

$$
W=\frac{1}{t} \sum_{k=1}^{t} W_{k}
$$

3.3. Total Hierarchical Sorting. Assuming that, in a decision index system with $r$ layers, the relative weight vector based on the $h$ th decision index is $P_{h}^{(r)}=\left[\begin{array}{llll}p_{1 h}^{(r)} & p_{2 h}^{(r)} & \cdots & p_{n_{r} h}^{(r)}\end{array}\right]^{T}$; when $h=1,2, \ldots, n_{r-1}$, the $n_{r} \times n_{r-1}$ order matrix is 
TABLE 1: Evaluation index system of old bridge reinforcement scheme.

\begin{tabular}{|c|c|c|}
\hline Decision set & Factor set & Index set \\
\hline \multirow{14}{*}{ Optimal selection of bridge reinforcement scheme $C$} & \multirow{5}{*}{ Structural functionality $S$} & Satisfaction of bearing capacity \\
\hline & & Satisfaction of durability \\
\hline & & Satisfaction of vibratility \\
\hline & & Satisfaction of safety \\
\hline & & Reinforcement cost \\
\hline & \multirow{4}{*}{ Economic rationality $E$} & Reinforcement period \\
\hline & & Subsequent maintenance cost \\
\hline & & Utilization degree of original structure \\
\hline & & Reliability of reinforcement technology \\
\hline & \multirow{3}{*}{ Technical feasibility $F$} & Applicability of reinforcement technology \\
\hline & & Complexity of reinforcement technology \\
\hline & & $\begin{array}{c}\text { Degree of impact of reinforcement on traffic } \\
\text { Structural aesthetics }\end{array}$ \\
\hline & \multirow{2}{*}{ Structural aesthetics $A$} & Environmental friendliness \\
\hline & & Environmental impact \\
\hline
\end{tabular}

$$
P^{(r)}=\left[\begin{array}{llll}
P_{1}^{(r)} & P_{2}^{(r)} & \ldots & P_{n_{r-1}}^{(r)}
\end{array}\right]^{T},
$$

where $p_{n h}^{(r)}$ is the relative weight vector based on the $h$ th decision index than the $h$ th decision.

If the comprehensive weight vector matrix of all decision indexes from the $r-1$ layer to the decision layer is $W^{(r-1)}=$ $\left[\begin{array}{llll}W_{1}^{(r-1)} & W_{2}^{(r-1)} & \ldots & W_{n_{r-1}}^{(r-1)}\end{array}\right]^{T}[16]$, then the comprehensive weight of all decision indexes from the $r$ layer to the decision layer is

$$
W^{(r)}=\left[\begin{array}{llll}
W_{1}^{(r)} & W_{2}^{(r)} & \ldots & W_{n_{r}}^{(r)}
\end{array}\right]^{T}=P^{(r)} W^{(r-1)} .
$$

Generally,

$$
W^{(r)}=P^{(r)} P^{(r-1)} \cdots P^{(3)} W^{(2)},
$$

where $W^{(2)}$ is actually the same as the relative weight vector under the single hierarchical sorting.

\section{Gray Relation Analysis Model}

It is difficult to obtain the optimal scheme by a gray system composed of known information and nondeterministic information. The gray relation analysis method [17] is a method to measure the relation degree among factors based on their similarity or dissimilarity in the development trend [18], which is suitable for solving the problem on multiobjective optimal selection decision-making of old bridge reinforcement design schemes.

4.1. Construction of Decision Matrix. Assuming that there are a total of $m$ old bridge reinforcement schemes and a total of $n$ decision indexes and $x_{i j}$ represents the attribute value of the $j$ th index of the $i$ th beam design scheme, then the initial decision matrix is

$$
X=\left[\begin{array}{cccc}
x_{11} & x_{12} & \ldots & x_{1 n} \\
x_{21} & x_{22} & \ldots & x_{2 n} \\
\ldots & \ldots & & \ldots \\
x_{m 1} & x_{m 2} & \ldots & x_{m n}
\end{array}\right]
$$

In the decision-making objectives of the old bridge reinforcement design schemes, for the decision index value corresponding to the bearing capacity, the higher is better, while for the decision index value corresponding to the economic indexes such as construction period and cost, the lower is better. Moreover, the different dimensions and orders of magnitude among decision indexes have a large impact on the evaluation and optimal selection of the beam design scheme. Therefore, in order to facilitate the gray relation analysis, all decision indexes of the beam design scheme are normalized [19].

4.1.1. Quantitative Index Processing. In the evaluation index system of old bridge reinforcement scheme, some evaluation indexes can be expressed by numerical values and directly used as quantitative indexes of the scheme, such as the reinforcement cost and construction period. Quantitative indexes can be processed as follows. For benefit indexes (the larger the attribute value is, the better), the dimensionless value is

$$
y_{i j}^{\prime}= \begin{cases}0, & y_{i j} \leq y_{i \min }, \\ \frac{y_{i j}-y_{i \min }}{y_{i \max }-y_{i \min }}, & y_{i \min }<y_{i j}<y_{i \max }, \\ 1, & y_{i j} \geq y_{i \max } .\end{cases}
$$

For cost indexes (the smaller the attribute value is, the better), the dimensionless value is

$$
y_{i j}^{\prime}= \begin{cases}0, & y_{i j} \geq y_{i \max }, \\ \frac{y_{i \max }-y_{i j}}{y_{i \max }-y_{i \min }}, & y_{i \min }<y_{i j}<y_{i \max }, \\ 1, & y_{i j} \leq y_{i \min },\end{cases}
$$

where $y_{i j}$ is the index value of the $i$ th index for the $j$ th scheme to be evaluated, $y_{i j}^{\prime}$ is the normalized value, $y_{i \text { min }}$ is 
the minimum index value in the $i$ th evaluation index of each scheme, i.e., $y_{i \min }=\min \left(\begin{array}{llll}y_{i 1} & y_{i 2} & \ldots & y_{i m}\end{array}\right)$, and $y_{i \max }$ is the maximum index value in the $i$ th evaluation index of each scheme, i.e., $\quad y_{i \max }=\max \left(\begin{array}{llll}y_{i 1} & y_{i 2} & \ldots & y_{i m}\end{array}\right)$, where $i=1,2,3, \ldots, n$ and $j=1,2,3, \ldots, m$.

4.1.2. Qualitative Index Processing. Some evaluation indexes in the evaluation index system of old bridge reinforcement scheme belong to qualitative indexes which can only be used for qualitative estimation and judgment. According to the needs of old bridge reinforcement, a 9level factor set [20] is adopted, $E=$ (worst, very poor, poor, relatively poor, medium, relatively good, good, very good, best), and the qualitative index language gray number is quantified by the linear gray number whitening weight function [21]. The quantification results are shown in Table 2.

4.2. Construction of Gray Relation Coefficient Matrix. Due to the relativity of bridge reinforcement scheme decision during comparison, an ideal reference scheme [22] is firstly constructed as $y_{0}=\left[\begin{array}{llll}y_{01} & y_{02} & \ldots & y_{0 n}\end{array}\right]$, where

$$
y_{0 j}=\max \left(y_{1 j}, y_{2 j}, \ldots, y_{m j}\right) \text {, }
$$

where $y_{m j}$ is the relative vector based on the $j$ th decision index than the $i$ th decision.

The ideal reference scheme can be understood as taking the best value of the corresponding evaluation index in all candidate design schemes as the reference sequence. The attribute values of decision indexes of $m$ design schemes are, respectively, taken as the comparison sequences, and the relation coefficient is used to measure the closeness of the data relationship between the reference sequence and the comparison sequence. The calculation formula of the relation coefficient of each decision index under different design schemes is

$$
\varepsilon_{i j}=\frac{\min _{i} \min _{j}\left|y_{0 j}-y_{i j}\right|+\rho \max _{i} \max _{j}\left|y_{0 j}-y_{i j}\right|}{\left|y_{0 j}-y_{i j}\right|+\rho \max _{i} \max _{j}\left|y_{0 j}-y_{i j}\right|},
$$

where $\varepsilon_{i j}$ is the relation coefficient between the $i$ th comparison sequence and the $j$ th index in the reference sequence $y_{0}, i=1,2, \ldots, m$ and $j=1,2, \ldots, n$, and $\rho$ is the identification coefficient $(\rho \in[0,1])$ and is generally taken as $\rho=0.5$.

4.3. Calculation of Gray Relation Degree. Combined with the fuzzy-AHP, the comprehensive weight vector matrix of all decision indexes at the third layer of the decision index system of the old bridge reinforcement scheme is determined as $W^{(3)}=\left[\begin{array}{llll}W_{1}^{(3)} & W_{2}^{(3)} & \ldots & W_{n 3}^{(3)}\end{array}\right]^{T}$, and the comprehensive weight vector of $n$ decision indexes to the decision layer in the decision index system is

$$
W^{(3)}=\left[\begin{array}{llll}
w_{1}^{(3)} & w_{2}^{(3)} & \ldots & w_{n}^{(3)}
\end{array}\right]^{T},
$$

where $w_{n}^{(3)}$ is the relative vector based on the $n$th decision index than all decision indexes at the third layer of the decision.

Let $w_{k}$ be the combined weight of the $k$ th index, and $\sum_{k=1}^{n} W_{k}^{3}=1$ [23]. So, the gray relation degree $\gamma_{i 0}$ between the old bridge reinforcement scheme and the ideal scheme is

$$
\gamma_{i 0}=\sum_{j=1}^{n} \varepsilon_{i j} w_{j}^{(3)}
$$

where $\varepsilon_{i j}$ is the calculation formula of the relation coefficient of each decision index under different design schemes and $w_{j}^{(3)}$ is the relative vector based on the $j$ th decision index than all decision indexes at the third layer of the decision.

4.4. Determination of Optimal Scheme. Firstly, according to the gray correlation degree, the evaluation schemes are sorted and optimized: the larger the relation degree is, the closer the reinforcement scheme is to the ideal scheme, and thus, the better the reinforcement scheme is, so as to determine the optimal scheme in the old bridge reinforcement schemes.

According to the gray relation axiom, gray relation degree $\gamma_{i 0}$ satisfies

(1) Normative $0<\gamma_{i 0} \leq 1, \gamma_{i 0}=1 \Leftarrow i=0$

(2) The smaller the proximity $|i-0|$, the greater the $\gamma(i, 0)$

Normativeness limits the value of gray correlation degree within the interval of $[0,1]$. Proximity indicates that the closer the two behaviors are, the more similar their changing trends are.

The evaluated schemes are sorted for optimal selection according to the gray relation degree: the larger the relation degree, the closer the reinforcement scheme is to the ideal scheme, and thus the better the reinforcement scheme, so as to determine the optimal scheme in the old bridge reinforcement schemes.

\section{Example of Decision-Making for Optimal Selection of Old Bridge Reinforcement Schemes}

5.1. General Situation and Reinforcement Schemes of Old Bridge. This paper takes the deck system reinforcement design schemes of a certain reinforced concrete ribbed arch bridge as an example, uses the fuzzy-AHP to determine the weight and gray relation analysis model for the optimal selection, and obtains the optimal reinforcement scheme, as well as verifies the effectiveness and practicability of the gray relation analysis method for the optimal selection of bridge reinforcement schemes based on fuzzy-AHP weights.

The bridge is a concrete-filled steel tube arch bridge, which was completed in July 1997, as shown in Figure 1. 
TABLE 2: Quantification results of qualitative index.

\begin{tabular}{lccccccrrr}
\hline Level & Worst & Very poor & Poor & Relatively poor & Medium & Relatively good & Good & Very good & Best \\
\hline Value & 0 & 0.125 & 0.250 & 0.375 & 0.500 & 0.625 & 0.750 & 0.875 & 1.000 \\
\hline
\end{tabular}

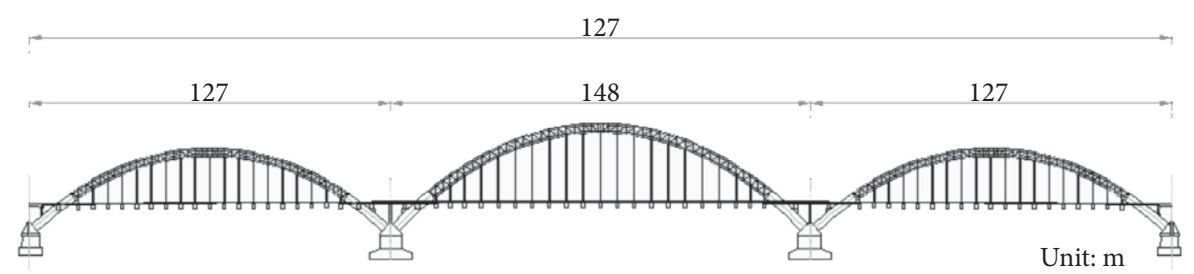

(a)

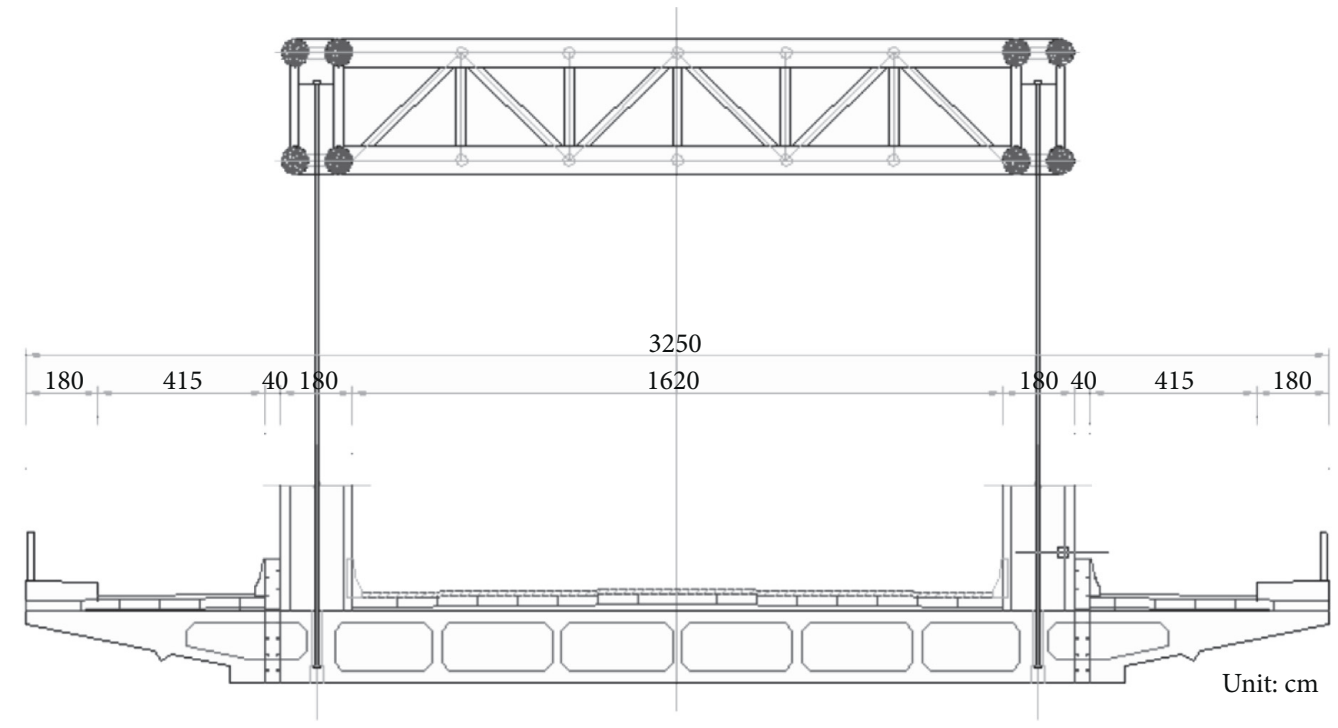

(b)

FIGURE 1: Bridge structure diagram. (a) Bridge elevation. (b) Bridge section view.

With the development of the city and the increase of traffic volume, the original design has approached the bearing capacity limit; the structure vibration is more obvious in actual use. Later, the bridge has been reinforced, with a hope to improve the dynamic characteristics and reduce the structural vibration response, but the reinforcement effect was not obvious. A total of four bridge deck reinforcement schemes are proposed this time: scheme 1 , replace the overall bridge deck; scheme 2, add longitudinal concrete beams; scheme 3, add longitudinal steel beams; scheme 4 , add longitudinal steel box-concrete composite beams, as shown in Figure 2.

\subsection{Determination of Comprehensive Weight of Decision} Indexes. As for determination on the relative weight of decision indexes at each layer of the old bridge reinforcement scheme, the pairwise comparison of structural functionality $S$, economic rationality $E$, technical feasibility $F$, and structural aesthetics $A$ are conducted according to the 0.1-0.9 scale method, to obtain the fuzzy complementary judgment matrix, see Table 3, for details.

According to formulas (1) and (2), the relative weight vector of the factor set is calculated as

$$
W_{1}=(0.308,0.242,0.258,0.192) \text {. }
$$

According to formulas (3) and (4), the compatibility index of $C_{1}$ and $S_{1}$ is obtained as $I\left(C_{1}, S_{1}\right)=0.104<0.2$, and the distribution of the relative weight vector $W_{1}$ of the corresponding objective layer is reasonable. Therefore, it is believed that the fuzzy judgment matrices are satisfactorily compatible. In conclusion, it is reasonable and reliable to use the mean value of the relative weight set as the relative weight vector of the objective layer. The relative weight vector of the objective layer is $W=(0.308,0.242,0.258$, 0.192).

Similarly, by constructing the fuzzy judgment matrix of the index layer, the relative weight vector of each decision index of the index layer is calculated, and thus, the comprehensive weight is calculated by formulas (6) (8), as shown in Table 4.

5.3. Calculation of Gray Relation Degree. According to formulas (9) (11), a decision matrix is established to select the optimal value of each index and determine the optimal scheme: 


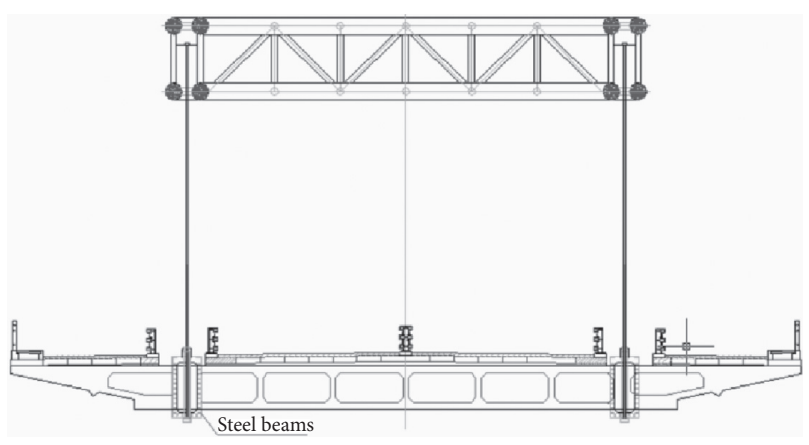

(a)

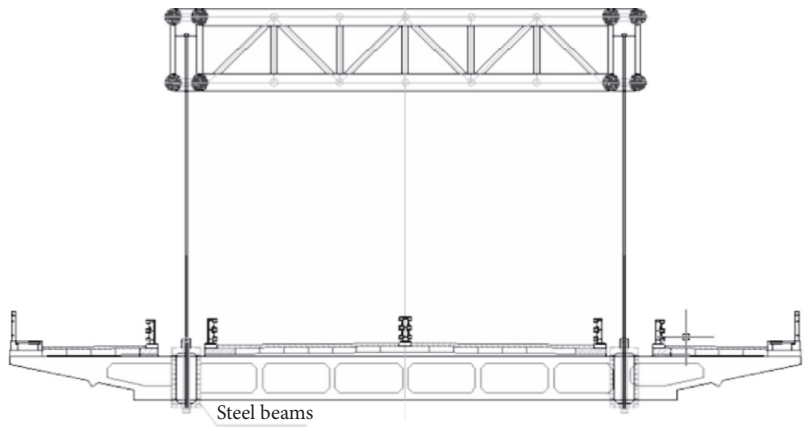

(c)

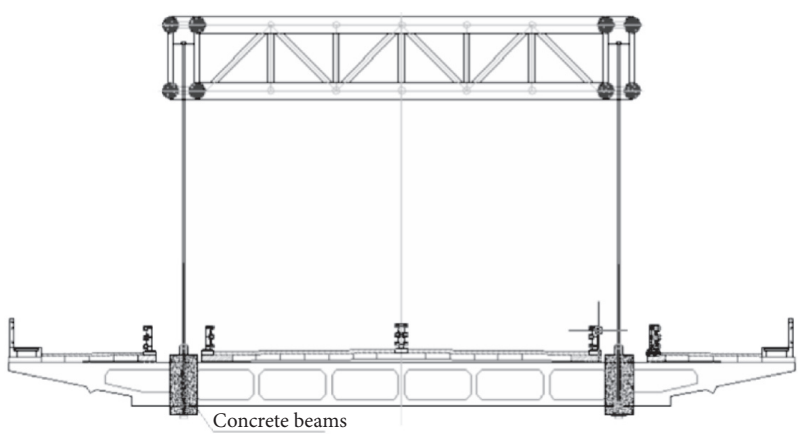

(b)

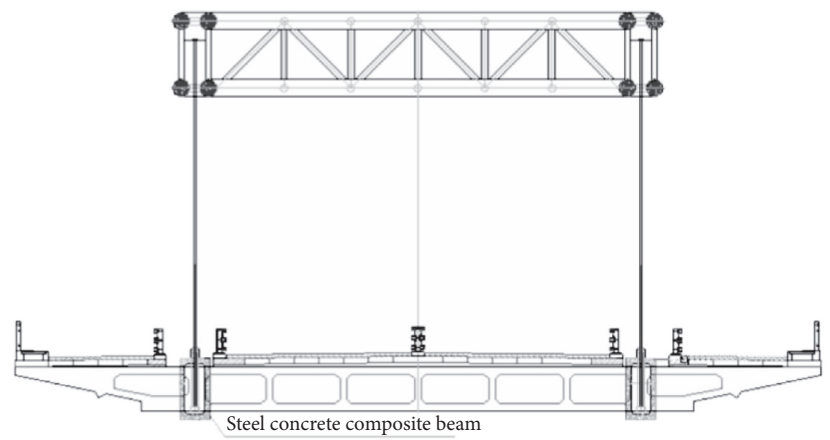

(d)

FiguRE 2: Bridge reinforcement schemes. (a) Scheme 1: replace the overall bridge deck. (b) Scheme 2: add longitudinal concrete beams. (c) Scheme 3: add longitudinal steel beams. (d) Scheme 4: add longitudinal steel box-concrete composite beams.

TABLE 3: Factor set importance evaluation matrix.

\begin{tabular}{lcccc}
\hline Factor set & Structural functionality $S$ & Economic rationality $E$ & Technical feasibility $F$ & Structural aesthetics $A$ \\
\hline Structural functionality $S$ & 0.5 & 0.7 & 0.7 & 0.8 \\
Economic rationality $E$ & 0.3 & 0.5 & 0.4 & 0.7 \\
Technical feasibility $F$ & 0.3 & 0.6 & 0.5 & 0.7 \\
Structural aesthetics $A$ & 0.2 & 0.3 & 0.3 & 0.5 \\
\hline
\end{tabular}

TABLE 4: Relative weights and comprehensive weights of decision indexes at various layers.

\begin{tabular}{|c|c|c|c|c|}
\hline Objective layer & Relative weight & Index layer & Relative weight & Comprehensive weight \\
\hline \multirow{4}{*}{ Structural functionality $S$} & \multirow{4}{*}{0.308} & Bearing capacity & 0.258 & 0.080 \\
\hline & & Durability & 0.208 & 0.064 \\
\hline & & Vibratility & 0.283 & 0.087 \\
\hline & & Safety & 0.250 & 0.077 \\
\hline \multirow{4}{*}{ Economic rationality $E$} & \multirow{4}{*}{0.242} & Reinforcement cost & 0.267 & 0.064 \\
\hline & & Reinforcement period & 0.275 & 0.066 \\
\hline & & Maintenance cost & 0.242 & 0.058 \\
\hline & & Utilization degree of original structure & 0.217 & 0.052 \\
\hline \multirow{4}{*}{ Technical feasibility $F$} & \multirow{4}{*}{0.258} & Reliability & 0.267 & 0.069 \\
\hline & & Practicality & 0.233 & 0.060 \\
\hline & & Complexity & 0.233 & 0.060 \\
\hline & & Traffic impact & 0.267 & 0.069 \\
\hline \multirow{4}{*}{ Structural aesthetic $A$} & \multirow{4}{*}{0.192} & Structural aesthetics & 0.267 & 0.051 \\
\hline & & Environmental friendliness & 0.233 & 0.045 \\
\hline & & Environmental impact & 0.233 & 0.045 \\
\hline & & Traffic impact & 0.267 & 0.051 \\
\hline
\end{tabular}




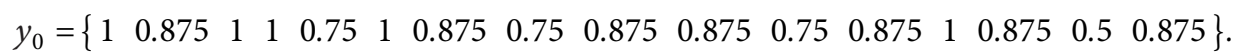

The gray relation coefficient matrix is calculated by formulas (12) and (13):

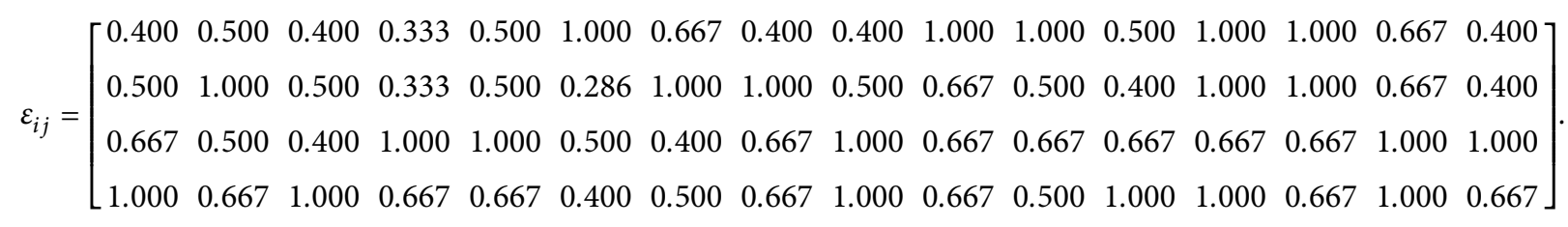

According to the comprehensive weight of decision index obtained in Table 4, the gray relation degree between each old bridge reinforcement scheme and the ideal optimal scheme is calculated by formulas (14) and (15):

$$
\begin{aligned}
& \gamma_{10}=0.612, \\
& \gamma_{20}=0.614, \\
& \gamma_{30}=0.708, \\
& \gamma_{40}=0.763 .
\end{aligned}
$$

It can be seen that the gray relation degree between scheme 4 and ideal optimal scheme is the largest, so scheme 4 "add longitudinal steel box-concrete composite beams" is the optimal scheme. The optimum selection result is consistent with the actual reinforcement scheme adopted.

\section{Conclusion}

(1) The fuzzy-AHP is used to construct the decision index system of the old bridge reinforcement scheme and determine the weight of the decision index, which makes the evaluation system more organized and systematic, and the index weight is more operable and quantitative, reducing the subjective evaluation impact and making the evaluation result more objective and reliable

(2) Fully considering the fuzzy and gray information of comparison and selection, the gray relation method is used for calculation and analysis of old bridge reinforcement schemes, thus selecting the optimal reinforcement scheme

(3) For the optimal selection of bridge reinforcement schemes, the gray relation analysis based on fuzzyAHP weights can select the optimal reinforcement scheme as a reference for the bridge reinforcement project and has a certain practical application value

\section{Data Availability}

All data, models, and codes generated or used during the study are included within the article.

\section{Conflicts of Interest}

The authors declare that they have no potential conflicts of interest with respect to the research, authorship, and/or publication of this article.

\section{Acknowledgments}

This work was supported by the Natural Science Foundation Projects of Liaoning Province (2019-ZD-0006 and 2019ZD-0145).

\section{References}

[1] Y. Kuo, T. Yang, and G.-W. Huang, “The use of grey relational analysis in solving multiple attribute decision-making problems," Computers \& Industrial Engineering, vol. 55, no. 1, pp. 80-93, 2008.

[2] M. Dağdeviren and İ. Yüksel, "Developing a fuzzy analytic hierarchy process (AHP) model for behavior-based safety management," Information Sciences, vol. 178, no. 6, pp. 1717-1733, 2008.

[3] H.-T. Nguyen, S. Z. M. Dawal, Y. Nukman, and H. Aoyama, "A hybrid approach for fuzzy multi-attribute decision making in machine tool selection with consideration of the interactions of attributes," Expert Systems With Applications, vol. 41, no. 6, pp. 3078-3090, 2014.

[4] H. F. Maghrabie, Y. Beauregard, and A. Schiffauerova, "Greybased multi-criteria decision analysis approach: addressing uncertainty at complex decision problems," Technological Forecasting and Social Change, vol. 146, pp. 366-379, 2019.

[5] V. Thakur and A. Ramesh, "Healthcare waste disposal strategy selection using grey-AHP approach," Benchmarking: An International Journal, vol. 24, no. 3, pp. 735-749, 2017.

[6] Z. Li and L. Chen, "A novel evidential FMEA method by integrating fuzzy belief structure and grey relational projection method," Engineering Applications of Artificial Intelligence, vol. 77, pp. 136-147, 2019.

[7] E. N. Kalemci, S. B. İkizler, T. Dede, and Z. Angın, "Design of reinforced concrete cantilever retaining wall using grey wolf optimization algorithm," Structures, vol. 23, pp. 245-253, 2020.

[8] A. Hafezalkotob, A. Hafezalkotob, H. Liao, and F. Herrera, "An overview of MULTIMOORA for multi-criteria decisionmaking: theory, developments, applications, and challenges," Information Fusion, vol. 51, pp. 145-177, 2019. 
[9] M. S. A. Khan and S. Abdullah, "Interval-valued pythagorean fuzzy GRA method for multiple-attribute decision making with incomplete weight information," International Journal of Intelligent Systems, vol. 33, no. 8, pp. 1689-1716, 2018.

[10] H. Naderpour, M. Mirrashid, and K. Nagai, "An innovative approach for bond strength modeling in FRP strip-toconcrete joints using adaptive neuro-fuzzy inference system," Engineering with Computers, vol. 36, no. 3, pp. 1083-1100, 2020.

[11] Z. Z. Wang and C. Chen, "Fuzzy comprehensive Bayesian network-based safety risk assessment for metro construction projects," Tunnelling and Underground Space Technology, vol. 70, pp. 330-342, 2017.

[12] J. L. Deng, "Introduction to Grey system theory," Journal of Grey System, vol. 1, no. 1, pp. 1-24, 1989.

[13] L. A. Zadeh, "Fuzzy sets as a basis for a theory of possibility," Fuzzy Sets and Systems, vol. 100, no. 1, pp. 9-34, 1999.

[14] H. D. Cheng, J.-R. Chen, C. Glazier et al., "Novel approach to pavement cracking detection based on fuzzy set theory," Journal of Computing in Civil Engineering, vol. 13, no. 4, pp. 270-280, 1999.

[15] Q. Zhou and V. V. Thai, "Fuzzy and grey theories in failure mode and effect analysis for tanker equipment failure prediction," Safety Science, vol. 83, pp. 74-79, 2016.

[16] A. M. Sefidian and N. Daneshpour, "Missing value imputation using a novel grey based fuzzy c-means, mutual information based feature selection, and regression model," Expert Systems With Applications, vol. 115, pp. 68-94, 2019.

[17] I. Bayane, A. Mankar, E. Brühwiler, and J. D. Sørensen, "Quantification of traffic and temperature effects on the fatigue safety of a reinforced-concrete bridge deck based on monitoring data," Engineering Structures, vol. 196, Article ID 109357, 2019.

[18] S. Liu, Y. Yang, N. Xie, and J. Forrest, "New progress of grey system theory in the new millennium," Grey Systems: Theory and Application, vol. 6, no. 1, pp. 2-31, 2016.

[19] H. Wang, Y.-M. Zhang, and Z. Yang, "A risk evaluation method to prioritize failure modes based on failure data and a combination of fuzzy sets theory and grey theory," Engineering Applications of Artificial Intelligence, vol. 82, pp. 216-225, 2019.

[20] M. Yazdani, C. Kahraman, P. Zarate, and S. C. Onar, "A fuzzy multi attribute decision framework with integration of QFD and grey relational analysis," Expert Systems With Applications, vol. 115, pp. 474-485, 2019.

[21] S. Sasmal, K. Ramanjaneyulu, S. Gopalakrishnan, and N. Lakshmanan, "Fuzzy logic based condition rating of existing reinforced concrete bridges," Journal of Performance of Constructed Facilities, vol. 20, no. 3, pp. 261-273, 2006.

[22] L. Di Sarno, C. Del Vecchio, G. Maddaloni, and A. Prota, "Experimental response of an existing RC bridge with smooth bars and preliminary numerical simulations," Engineering Structures, vol. 136, pp. 355-368, 2017.

[23] J. Xue, P. H. A. J. M. Van Gelder, G. Reniers, E. Papadimitriou, and $\mathrm{C} . \mathrm{Wu}$, "Multi-attribute decision-making method for prioritizing maritime traffic safety influencing factors of autonomous ships' maneuvering decisions using grey and fuzzy theories," Safety Science, vol. 120, pp. 323-340, 2019. 